\title{
Projeto UCA-Assistiva: mapeamento e avaliação de ferramentas assistivas nos laptops educacionais do PROUCA
}

Ana Grasielle Dionísio Corrêa ${ }^{1}$, Patrícia Araújo de Oliveira ${ }^{1}$, Gilda Aparecida de Assis $^{1}$, Irene Karaguilla Ficheman ${ }^{1}$, Marilena do Nascimento ${ }^{2}$, Shirley Takeco Gobara $^{3}$, Elda Gomes Araújo ${ }^{4}$, Roseli de Deus Lopes ${ }^{1}$

${ }^{1}$ Laboratório de Sistemas Integráveis da Escola Politécnica (LSI-EP)

Universidade de São Paulo (USP)

Av. Prof. Luciano Gualberto, travessa 3, nº 158, CEP: 05508-970, São Paulo/SP, Brasil

${ }^{2}$ Colmeia Espaço Terapêutico de Medicina Integrada R. Itararé, 75, Bela Vista, CEP: 01308-030, São Paulo/SP, Brasil

${ }^{3}$ Departamento de Física da Universidade Federal de Mato Grosso do Sul (UFMS) Av. Costa e Silva s/n, Cidade Universitária, CEP: 79070900, Campo Grande/MS, Brasil

${ }^{4}$ Colegiado de Pedagogia da Universidade Federal do Amapá (UNIFAP) Rodovia JK Zerao, CEP: 68900-000, Macapa/AP, Brasil

Resumo. Este artigo apresenta resultados do mapeamento e avaliação de ferramentas assistivas nos laptops educacionais do projeto UCA. Como resultado pôde-se obter as vantagens e desvantagens da utilização de uma série de ferramentas de acessibilidade segundo critérios de um especialista em Tecnologia Assistiva. Para isso, foi realizado um mapeamento de diversas ferramentas que foram elencadas por especialistas em acessibilidade, as mais votadas foram selecionadas e testadas no laptop Classmate PC para verificar sua viabilidade de utilização.

Palavras-chaves: tecnologias assistivas, pessoas com deficiência, projeto UCA.

Abstract. This paper presents the results of the mapping and evaluation of assistive tools in educational laptops of the project UCA. As the result it was possible to obtain the advantages and disadvantages of using a set of tools accessibility according to criteria of a expert on Assistive Technology. For this it was conducted one mapping of several tools that were listed by accessibility experts, the Highest Rated were selected and tested in laptop Classmate PC to check its feasibility of using.

Keywords: assistive technologies, people with disabilities, UCA project. 


\section{Introdução}

O Projeto Um Computador por Aluno (PROUCA), desde sua implantação no Brasil, em 2007, tem investido esforços para distribuir laptops educacionais nas escolas e capacitar coordenadores e professores para o trabalho em sala de aula (Schaeffer et al., 2012), (Spagnolo et al., 2012), (Venâncio et al., 2012), (Gomes, 2008). Também, em 2007, o Governo brasileiro instaurou o Decreto 6.094 que estabelece, nas diretrizes do Compromisso Todos pela Educação, a garantia do acesso e permanência no ensino regular e o atendimento às necessidades educacionais especiais dos alunos, fortalecendo seu ingresso nas escolas públicas. Desde então, várias pesquisas sobre acessibilidade para pessoas com deficiência podem ser encontradas na literatura (Filho, 2008).

De acordo com o Decreto 3298/99 que regulamenta a Lei 7853/89, considera-se deficiência toda perda ou anormalidade de uma estrutura ou função psicológica, fisiológica ou anatômica que gere incapacidade para o desempenho de atividade, dentro do padrão considerado normal para o ser humano. Segundo o Decreto ${ }^{0} 5296$, existem cinco tipos de deficiência, são elas: física, auditiva, visual, mental e múltipla. De acordo com o último censo demográfico de 2010 realizado pelo Instituto Brasileiro de Geografia e Estatística (IBGE), a deficiência visual é a de maior incidência de casos no Brasil (35,7 milhões), seguida pela deficiência motora (13,3 milhões), auditiva (9,7 milhões) e mental ou intelectual (2,6 milhões).

Visando reduzir os problemas funcionais das pessoas com deficiência, são utilizados equipamentos, serviços, estratégias e práticas denominadas de Tecnologias Assistivas (Cook e Hussey, 1995). De acordo com Bersch (2006) Tecnologias Assistivas (TAs) são categorizadas por diversas perspectivas, tais como: auxílios para a vida diária; comunicação aumentativa e alternativa; recursos de acessibilidade ao computador; sistemas de controle de ambiente; projetos arquitetônicos para acessibilidade; órteses e próteses; adequação postural; entre outros. No âmbito do projeto UCA-Assistiva, foram consideradas para estudo as categorias de adaptação física (órteses e próteses) e recursos de acessibilidade ao computador, que incluem: equipamentos de entrada e saída (síntese de voz e Braille), auxílios alternativos de acesso (ponteiras de cabeça), teclados modificados ou alternativos, acionadores, softwares especiais que possibilitam que pessoas com deficiência possam utilizar o computador.

Pesquisas envolvendo a acessibilidade e inclusão das crianças com necessidades especiais com uso dos laptops educacionais do PROUCA ainda são escassos. Canal, Almeida e Baranauskas (2012), por exemplo, realizaram uma pesquisa sobre a dislexia e suas implicações no uso das aplicações do laptop XO. Contudo, estudos sobre o uso das Tecnologias Assistivas nos laptops educacionais, em especial os laptops Classmate PC, para apoio a crianças com outras deficiências precisam também ser contemplados. Por este motivo, este trabalho vem contribuir com uma pesquisa sobre a avaliação de ferramentas de acessibilidade nos laptops educacionais Classmate PC que possam ampliar as possibilidades de uso por crianças com deficiência motora, visual e auditiva.

\section{Fundamentação Teórica}

O termo “Assistive Technology”, traduzido no Brasil como “Tecnologia Assistiva”, faz parte da legislação norte-americana desde 1998. A definição legal, nos EUA, para o termo é: "qualquer item, peça de equipamento ou produto, podendo ser adquirido 
comercialmente, modificado ou personalizado, usado para aumentar, manter ou melhorar capacidades funcionais de indivíduos com deficiência" (Cook e Polgar, 2008).

Na legislação brasileira também foi previsto explorar o assunto, inicialmente concebido com o nome de Ajudas Técnicas. Desde então, as expressões "Tecnologia Assistiva", "Ajudas Técnicas" e "Tecnologia de Apoio" vêm sendo utilizadas como sinônimos. O Comitê de Ajudas Técnicas propõe o seguinte conceito para Tecnologias Assistivas (BRASIL, 2013): "área do conhecimento, de característica interdisciplinar, que engloba produtos, recursos, metodologias, estratégias, práticas e serviços que objetivam promover a funcionalidade, relacionada à atividade e participação de pessoas com deficiência, incapacidade ou mobilidade reduzida, visando sua autonomia, independência, qualidade de vida e inclusão social".

O Ministério da Educação do Brasil lançou, em 2002, o Portal de Ajudas Técnicas com o objetivo de apoiar a escola e profissionais da educação para viabilizar e melhorar sua prática profissional. O Portal disponibiliza fascículos com diretrizes para confecção e uso de materiais acessíveis que facilitem a aprendizagem dos alunos com deficiência no que se refere a: recursos pedagógicos adaptados; adaptadores manuais; informática; mobiliário adaptado; mobilidade; recursos para comunicação alternativa.

Bersch e Tonolli (2010) classificam as Tecnologias Assistivas segundo as diretrizes gerais da Americans With Disabilities Act (ADA). Entretanto, esta classificação não é definitiva e pode variar segundo alguns autores. A importância dessa classificação é que possibilita organizar a utilização, prescrição, estudo e pesquisa dos materiais assistivos, além de oferecer ao mercado focos específicos de trabalho e especialização. Dessa forma, tem-se a seguinte classificação:

1. Auxílios para a vida diária: materiais e produtos para auxílio em tarefas rotineiras tais como comer, cozinhar, vestir-se, tomar banho e executar necessidades pessoais, manutenção da casa etc.

2. Comunicação aumentativa e alternativa: recursos, eletrônicos ou não que permitem a comunicação expressiva e receptiva das pessoas sem a fala ou com limitações da mesma. São muito utilizadas as pranchas de comunicação como sistema Bliss de comunicação e o Picture Communication Symbols (PCs). O Bliss é um sistema simbólico gráfico visual capaz de representar conceitos abstratos. O PCs é um sistema gráfico visual que contém desenhos simples, podendo-se acrescentar, na medida do necessário, fotografias, figuras, números, círculos para as cores, o alfabeto, outros desenhos ou conjuntos de símbolos.

3. Recursos de acessibilidade ao computador: equipamentos de entrada e saída (síntese de voz, Braille), auxílios alternativos de acesso (ponteiras de cabeça), teclados modificados ou alternativos, acionadores, softwares especiais (de reconhecimento de voz, etc.).

4. Sistemas de controle de ambiente: sistemas eletrônicos que permitem às pessoas com limitações moto-locomotoras, controlar remotamente aparelhos eletroeletrônicos, sistemas de segurança, entre outros, localizados em seu quarto, sala, escritório, casa e arredores. 
5. Projetos arquitetônicos para acessibilidade: adaptações estruturais e reformas na casa e/ou ambiente de trabalho, através de rampas, elevadores, adaptações em banheiros entre outras, que retiram ou reduzem as barreiras físicas, facilitando a locomoção da pessoa com deficiência.

6. Órteses e próteses: troca ou ajuste de partes do corpo, faltantes ou de funcionamento comprometido, por membros artificiais ou outros recurso ortopédicos (talas, apoios etc.). Incluem-se os protéticos para auxiliar nos déficits ou limitações cognitivas, como os gravadores de fita magnética ou digital que funcionam como lembretes instantâneos.

7. Adequação Postural: adaptações para cadeira de rodas visando o conforto e distribuição adequada da pressão na superfície da pele (almofadas especiais, assentos e encostos anatômicos), bem como posicionadores e contentores que propiciam maior estabilidade e postura adequada do corpo através do suporte e posicionamento de tronco, cabeça e membros.

8. Auxílios de mobilidade: cadeiras de rodas manuais e elétricas, bases móveis, andadores, scooters de três rodas e qualquer outro veículo utilizado na melhoria da mobilidade pessoal.

9. Auxílios para cegos ou com visão sub-normal: auxílios para grupos específicos que inclui lupas e lentes, Braille para equipamentos com síntese de voz, grandes telas de impressão, sistema de TV com aumento para leitura de documentos, publicações, entre outros.

10. Auxílios para surdos ou com déficit auditivo: auxílios que incluem vários equipamentos (infravermelho, FM), aparelhos para surdez, telefones com teclado teletipo, sistemas com alerta táctil-visual, entre outros.

11. Adaptações em veículos: acessórios e adaptações que possibilitam a condução do veículo, elevadores para cadeiras de rodas, camionetas modificadas e outros veículos automotores usados no transporte pessoal.

A importância das classificações no âmbito das Tecnologias Assistivas se dá pela promoção da organização desta área de conhecimento e possibilita servir de estudo, pesquisa, desenvolvimento, promoção de políticas públicas, organização de serviços, catalogação e formação de banco de dados para identificação dos recursos mais apropriados ao atendimento de uma necessidade funcional do usuário final.

No do projeto UCA-Assistiva, são consideradas as categorias: 2) comunicação aumentativa e alternativa; 3) recursos de acessibilidade ao computador; 9) auxílios para cegos ou com visão subnormal; 10) auxílios para surdos ou com déficit auditivo.

\section{Materiais e Métodos}

Este projeto, intitulado UCA-Assistiva, está dividido em cinco etapas:

Na Etapa I foi feito um levantamento das crianças com deficiência nas escolas envolvidas neste projeto e de suas necessidades de acessibilidade nos laptops educacionais. 
Na Etapa II foi feito um mapeamento das tecnologias de hardware e software de acessibilidade livres existentes no mercado e em pesquisas científicas.

Na Etapa III foram realizados testes funcionais com as tecnologias livres elencadas na Etapa II nos laptops Classmate PC com vista a possibilitar seu uso imediato nas escolas participantes do projeto.

Na Etapa IV foi realizado um estudo sobre a viabilidade técnica de adequações, melhorias e/ou desenvolvimento de tecnologias livres que atendam às necessidades levantadas na Etapa I. Foram propostas soluções de hardware e software livres para desenvolvimento.

A Etapa V destina-se à divulgação dos resultados desta pesquisa.

Este artigo aborda os resultados obtidos nas etapas II e III onde foram feitos o mapeamento, testes e avaliações das tecnologias livres elencadas para a pesquisa. Esses resultados estão descritos na próxima seção.

\section{Resultados e Discussões}

Para a execução da Etapa II, primeiramente foi necessário realizar um estudo sobre os Sistemas Operacionais (SOs) compatíveis com o Classmate PC. Isso porque o Metasys, SO originalmente instalado no Classmate PC não possibilitou a instalação de recursos de acessibilidade. Os resultados são apresentados a seguir.

\subsection{Sistemas Operacionais Compatíveis com o Classmate PC}

O equipamento adquirido para a fase piloto do UCA foi o Classmate PC, computador móvel de baixo custo que pode operar em ambientes de rede com ou sem fio. $\mathrm{O}$ Classmate PC permite que estudantes colaborem, troquem informações e revisem materiais de aprendizado. Permite também que professores suplementem e ampliem suas aulas, fornecendo características para o controle dos alunos (Schneider, Santarosa e Conforto, 2011).

O Classmate PC, desenvolvido nacionalmente pela CCE-Info, pesa 1,3 kg e sua configuração inclui processador Intel Atom N270, relógio de 1.6 gigaHertz, capacidade de armazenamento de 4 GB em cartão SD, memória principal de 512 megabytes, tela de cristal líquido de sete polegadas, placa de som com entrada para microfone, microfone embutido e duas caixas acústicas, webcam de 0.3 megapixels (Digibras 2013).

O Classmate PC é atualmente distribuído com o SO Metasys Classmate que é baseado no SO Metasys, com algumas alterações específicas para a plataforma Classmate PC (Schneider, Santarosa e Conforto, 2011). O Metasys Classmate inclui alguns aplicativos gerais, tais como Internet, escritório e multimídia, e outros e aplicativos específicos para o desenvolvimento de atividades educacionais, tais como KTurtle e Squeak (ambientes de programação), Tux Typing (digitação para crianças), Tux Math (operações matemáticas), entre outros (International Syst S/A 2013).

Apesar do uso eficaz do Metasys Classmate, este SO se mostrou limitado em termos de capacidades de instalação, suporte e funcionamento adequado dos recursos de acessibilidade, pois nenhuma das ferramentas de acessiblidade elencadas (Seção 4.2) foi instalada com êxito. Dessa forma, este estudo buscou a avaliação de outros SOs livres que fossem compatíveis com a plataforma Classmate PC, são eles: 
Lubuntu: também chamado de Light Ubuntu, desenvolvido para computadores móveis, é mais compacto, exige menos recursos de hardware e tem uma melhor eficiência energética do que as outras distribuições do Ubuntu.

Linux Acessível: trata-se de uma distribuição Linux direcionada especificamente para pessoas com deficiência visual e tem como principal objetivo o de adicionar, corrigir e configurar os recursos de acessibilidade e usabilidade para pessoas com esta deficiência.

UbuntUCA: trata-se de uma remasterização do Ubuntu, desenvolvida para o uso em netbooks (como os laptops UCA) e no ambiente educacional. Sua interface é adaptada para telas pequenas, além de ser agradável, compacto e funcional. Sua coleção de aplicativos é capaz de atender às principais necessidades dos professores e alunos em sala de aula, as quais foram levantadas no âmbito do projeto durante dois Workshops com professores do projeto UCA. As atividades pedagógicas mais citadas pelos professores participantes dos Workshops foram: Pesquisa de informações via Internet; Escrita de texto; Destaque de texto; Desenho; Cálculo matemático; Apresentação; Vídeo.

A Tabela 1 apresenta uma comparação entre os quatro SOs testados no Classmate PC em termos de memória e capacidade de armazenamento.

Tabela 1. Sistema Operacional versus Ocupação de Memória

\begin{tabular}{|c|c|c|c|}
\hline SO & RAM 'livre' & $\begin{array}{c}\text { Armazenamento do } \\
\text { SO }\end{array}$ & $\begin{array}{c}\text { Armazenamento } \\
\text { disponível }\end{array}$ \\
\hline Lubuntu & $400 \mathrm{MB}$ & $3 \mathrm{~GB}$ & $900 \mathrm{MB}$ \\
\hline Linux Acessível & $350 \mathrm{MB}$ & $3 \mathrm{~GB}$ & $900 \mathrm{MB}$ \\
\hline UbuntUCA & $500 \mathrm{MB}$ & $3 \mathrm{~GB}$ & $1 \mathrm{~GB}$ \\
\hline
\end{tabular}

As opções de SO Lubuntu e Linux acessível se mostraram as mais adequadas para este projeto. Optou-se pelo Linux acessível, pois o aplicativo de acessibilidade Orca já vem instalado como recurso de acessibilidade padrão (RENAPI 2012). Trata-se de um leitor de telas capaz de interagir com o SO do computador capturando toda e qualquer informação apresentada em forma textual, transformando-a em uma resposta falada através de um sintetizador de voz. Além de ser um leitor de telas, o aplicativo também possibilita ampliar textos e imagens, permitindo aos usuários com baixa visão, a utilização de lupas para ampliação e melhor visualização da tela.

\subsection{Levantamento e Viabilidade de Ferramentas de Acessibilidade}

Foi feito um levantamento dos recursos de acessibilidade que auxiliam pessoas com deficiência física, visual, cognitiva e/ou auditiva e se enquadram em um dos grupos classificados por Galvão Filho e Damasceno (2008): (1) adaptações físicas ou órteses, (2) adaptações de hardware e (3) adaptações de software. Foram selecionados 89 recursos através de pesquisa na literatura, pesquisas na Internet e consultas aos especialistas com experiência comprovada na interação com pessoas com deficiência.

O levantamento obtido foi apresentado, na forma de questionário, a um total de 13 especialistas em acessibilidade, com especialização em um ou mais tipos de deficiências e que atuam no atendimento de crianças ou adultos em instituições de 
tratamento ou apoio pedagógico de inclusão. Esses especialistas opinaram sobre cada produto, qualificando-o segundo uma escala de frequência verbal (ótimo, regular, ruim, não conheço). Os especialistas também opinaram sobre o valor de aquisição do produto. A partir dos resultados da votação, foi criado um ranking dos produtos mais votados e, a partir desse ranking, foi feita uma verificação da viabilidade de utilização desses produtos nos laptops Classmate PC.

A principal dificuldade de utilização desses recursos de acessibilidade foi o fato de que muitos dos softwares elencados são compatíveis apenas com Sistemas Operacionais proprietários ou são softwares proprietários ou são recursos de hardware. Dessa forma, foi necessário buscar softwares que fossem semelhantes aos indicados no ranking. A Tabela 2 mostra os recursos mais votados e a opção equivalente adotada.

Tabela 2. Softwares mais votados por especialistas e as alternativas adotadas

\begin{tabular}{|l|l|l|}
\hline Software & $=>$ & Alternativa encontrada \\
\hline $\begin{array}{l}\text { Opções de acessibilidade do Windows } \\
\text { XP }\end{array}$ & $=>$ & $\begin{array}{l}\text { Pacote de softwares de acessibilidade do Linux } \\
\text { Acessível }\end{array}$ \\
\hline Mouse Ocular & $=>$ & Eviacam \\
\hline Magic ampliador de ecrã & $=>$ & Ampliador do Orca \\
\hline Rata Virtual & $=>$ & Gok-Gnome \\
\hline MPB - mouse por barrido & $=>$ & Gok-Gnome \\
\hline Meta Mouse & $=>$ & Ampliador do Orca \\
\hline ReadPlease & $=>$ & Leitor do Orca \\
\hline Falador & $=>$ & Leitor do Orca \\
\hline Teclado amigo & $=>$ & Gok-Gnome \\
\hline Dosvox & $=>$ & Leitor do Orca \\
\hline Jaws & $=>$ & Leitor doOrca \\
\hline Windows eyes & $=>$ & Leitor do Orca \\
\hline Editor amigo & $=>$ & Gok-Gnome e editor de texto do linux acessível \\
\hline Grid & $=>$ & Gok-Gnome \\
\hline Headdev & $=>$ & Eviacam \\
\hline
\end{tabular}

Algumas das alternativas adotadas apresentaram vantagens em relação à compatibilidade com o SO Linux Acessível, como por exemplo: a) Eviacam que é um software de código livre para rastreamento de cabeça, ou seja, por meio de uma webcam, possibilita substituir o mouse por movimentos da cabeça. O Eviacam possui a vantagem de ser compacto e é considerado eficiente pelos especialistas, pois dificilmente perde a calibração; b) Gok-Gnome que possui funcionalidades como teclado e mouse virtuais; c) Outras ferramentas possuem a vantagem de já estarem disponíveis no Linux Acessível, como os pacote de softwares de acessibilidade do Linux Acessível e o Orca (leitor de tela, lupa, contraste, tamanho da fonte). 


\subsection{Teste com as ferramentas}

Foram utilizados 4 laptops Classmate PC com o SO escolhido (Linux Acessível) e os softwares de acessibilidade elencados (Eviacam, Gok-Gnome e Orca). A testificação foi realizada com uma especialista em Tecnologia Assistiva que assiste crianças com deficiência em Centros de Tratamento especializados. Foi desenvolvido um manual de manuseio do Classmate PC e dos recursos de acessibilidade nele instalados visando facilitar o entendimento por parte da especialista. O manual continha uma descrição das ferramentas de acessibilidade instaladas, descrição de como acessá-las e propostas de tarefas pedagógicas a serem desenvolvidas nas escolas. A definição dessas tarefas foi feita por meio de uma pesquisa bibliográfica sobre a utilização dos laptops no PROUCA e de entrevistas com professores de algumas escolas contempladas com o projeto UCA.

Ao utilizar a ferramenta, a especialista argumentava sobre os pontos positivos e negativos observados e, a partir das suas observações, foram identificados alguns requisitos já atendidos ou parcialmente atendidos ou ainda não atendidos pelas mesmas e que precisaam ser considerados em futuras versões. A partir de visitas para realização de testificações, foram levantadas algumas questões para adequações e/ou adaptações.

Nos testes, percebeu-se que a especialista não conseguiu utilizar o teclado virtual Gok-Gnome executando uma atividade de escrever uma carta, por exemplo, pois o teclado virtual ocupou uma parte significativa da tela. Dessa forma, o texto da carta, que estava sendo redigida, ficava atrás do teclado, impossibilitando a visualização do que estava sendo digitado. Esse problema se manteve mesmo diminuindo o tamanho da janela. Dessa forma, houve a necessidade de buscar alternativas de teclados virtuais que ocupassem menos espaço da tela. Foi então elencado o teclado XVKBD, pois ele ocupa apenas uma faixa da tela e não apresentou outro tipo de problema na testificação.

Após o teste com teclado virtual, a especialista sugeriu que houvesse também o feedback auditivo, além do visual, para que o usuário pudesse ler e também escutar o que está sendo digitado. Outra forma de uso do teclado virtual sugerida foi a sua utilização juntamente com o Eviacam, ou seja, o usuário utiliza o mouse comandado pelo Eviacam para digitar um texto usando o teclado virtual.

Nos testes realizados com o Eviacam, a especialista alertou sobre um problema: o software não possibilita congelar o mouse somente em um eixo. Por exemplo, ao congelar no eixo x, o mouse se moveria somente na horizontal. Isso é importante, segundo a especialista, para usuários que tem espasmos (contração involuntária de um músculo), o que dificulta o manuseio do mouse.

Foram identificados dois problemas principais na utilização da ferramenta leitor de tela Orca. O primeiro é o não funcionamento do mesmo o software de desenho Tuxpaint, pois ele não lia as opções do software. Foram testados outros softwares: RGBpaint, Kolour Paint, GNU Paint, Xpaint, e o leitor de tela não funcionou de forma satisfatória em nenhum deles. O leitor também não lê os botões da ferramenta de desenho e o usuário não tem a informação da localização do cursor para desenhar. No entanto, a especialista frisou que não há necessidade do leitor de tela ser compatível com o software de desenho, pois essa atividade não pode ser executada por cegos e no caso de pessoas com baixa visão o ampliador de tela já é um recurso que pode ajudar. 
Outro problema identificado é o fato do leitor não ler as teclas Shift, TAB, Caps_Lock. Nesse caso, foi inserida uma marca tátil nas teclas para identificá-las.

Outra necessidade levantada pela especialista foi utilização de um software que identificasse comandos de voz, porém nenhum programa foi instalado com êxito no laptop Classmate PC. Tais softwares apresentaram problema como, por exemplo, o travamento do Sistema Operacional, provavelmente por este possuir recursos de hardware limitados. Foram encontrados outros softwares de detecção de voz que necessitavam de conhecimentos mais específicos para sua utilização, como a necessidade de programação. Dessa forma, a testificação desse tipo de ferramenta não foi possível e foi descartada sua utilização no laptop Classmate PC.

Para algumas propostas de atividades pedagógicas se faz necessária a utilização de um software de vídeo. Dessa forma, houve um levantamento de qual seria a opção que fosse compatível com o Linux e funcionasse de forma satisfatória. Foi escolhido o Cheese, que é largamente recomendado em pesquisas na Internet. O Cheese apresentou alguns problemas como demora em começar a gravação e a não continuidade da gravação, com a impressão de travamento. A solução encontrada foi a diminuição da qualidade do vídeo. Esta configuração foi inserida no manual.

\section{Conclusões}

Com o intuito de fornecer subsídios para a inclusão de crianças com deficiência nas escolas UCA, este projeto teve por objetivo levantar e testar ferramentas acessíveis livres para os laptops Classmate PC. Observou-se que a proposta inicial do Projeto UCA, que é de disponibilizar um laptop de baixo custo para cada aluno da unidade escolar, não atendeu às crianças com deficiência, pois lhe faltavam ferramentas de interação com os laptops e essas crianças vêm sendo excluídas das atividades pedagógicas conduzidas com os laptops nas escolas.

Houve o levantamento de ferramentas acessíveis e foi identificada a viabilidade de utilização dessas ferramentas nos laptops através de auxilio de especialistas. Com os resultados parciais alcançados, é possível afirmar que as ferramentas escolhidas ajudarão algumas crianças com deficiência na realização de atividades pedagógicas.

Como trabalhos futuros, pretende-se relatar os resultados dos testes que estão sendo conduzidos com as crianças com deficiência nas escolas, acompanhadas por professores nos Estados de São Paulo (SP), Mato Grosso do Sul (MS) e Amapá (AP), onde serão verificadas a aplicabilidade das ferramentas no ambiente escolar. Espera-se obter resultados promissores para que seja possível contemplar e incluir todas as crianças que frequentam as escolas.

\section{Referências}

BERSCH, R. Tecnologia assistiva e educação inclusiva. In: Ensaios Pedagógicos, Brasília: SEESP/MEC, p. 89-94, 2006.

CANAL, M.C.; ALMEIDA, L.D. e BARANAUSKAS, M.C.C Uma avaliação de acessibilidade no laptop educacional da OLPC na perspectiva de pessoas com dislexia. Anais do $\mathbf{2 3}^{\circ}$ Simpósio Brasileiro de Informática na Educação (SBIE), 2012. 
COOK e HUSSEY. Assistive Tecnologies: Principles and Pratice, Mosby. Year Book, USA-Missouri, 1995.

DIGIBRAS - Manual de Instruções do Laptop Educacional. Disponível em $<$ http://download.cceinfo.com.br/manuais/CM52C.pdf>; Acesso em 21 de out. 2013.

FILHO, G.; DAMASCENO, L. Tecnologia Assistiva em Ambiente Computacional: Recursos para a Autonomia e Inclusão Sócio-Digital da Pessoa com Deficiência. In: Instituto de Tecnologia Social - ITS. (Org.), Tecnologia Assistiva nas Escolas: Recursos Básicos de Acessibilidade Sócio-Digital para Pessoas com Deficiência, 1ed., São Paulo: ITS, v. 1, 2008, pp. 25-38.

GOMES, A. Um computador por aluno: a experiência brasileira. In: RELATOR P. H. LUSTOSA, Equipe técnica LOPES, C.A.; QUEIROZ FILHO, A.P.; CAMELO, A.L. Brasília: Câmara dos Deputados, Coordenação de Publicações, 2008, p. 195 (Série avaliação de políticas públicas 1).

RENAPI - Rede Nacional de Pesquisa e inovação em Tecnologias Digitais. Ministério da Educação - Secretaria de Educação Profissional e Tecnológica Rede Nacional de Pesquisa e Inovação em Tecnologias Digitais Projeto de Acessibilidade Virtual. Disponível em: http://acessibilidade.bento.ifrs.edu.br/arquivos/pdf/manual/manual-02-arquivo-26.pdf.

Acesso em 22 de outubro de 2013.

SCHAEFFER, A.G.; BEAUTLER, D.; GOTTARDO, E. Linux como sistema operacional em computadores do programa UCA. In: Anais do 10 Seminário Nacional de Inclusão Digital (SENID). Disponível em: http://senid.upf.br/2012/anais/96137.pdf. Acesso em: 20 de out. de 2012.

SPAGNOLO, C.; COPINI, I.B.; ZACHARIAS, L.C.; BASSANI, E.L.; ECO, R.M.R.; RIGO, S.B.; TEDESCO, S. Formação de Professores - PROUCA: oportunidades e desafios para docentes. In: Anais do 1o Seminário Nacional de Inclusão Digital (SENID). Disponível em: http://senid.upf.br/2012/anais/96154.pdf. Acesso em: 20 de outubro de 2012.

VENANCIO, V.; CORREA, A.G.D.; TELLES, E.O.; CORREA, C.M.; GARCIA, B.V.R.; RIBEIRO, C.C.; FICHEMAN, I.K.; LOPES, R.D. Integração dos Laptops Educacionais ao Cotidiano Escolar no UCA São Paulo: facilidades e dificuldades. In: I Congresso Brasileiro de Recursos Digitais na Educação, São Paulo, 2012.

SCHNEIDER, F. C.; SANTAROSA, L. M. C. e CONFORTO, D. Cidade Um Computador por Aluno - UCA Total A identificação de situações inclusivas na totalidade In: Simpósio Brasileiro de Informática na Educação (SBIE), 2011, Aracajú. Anais do SBIE, 2011. 